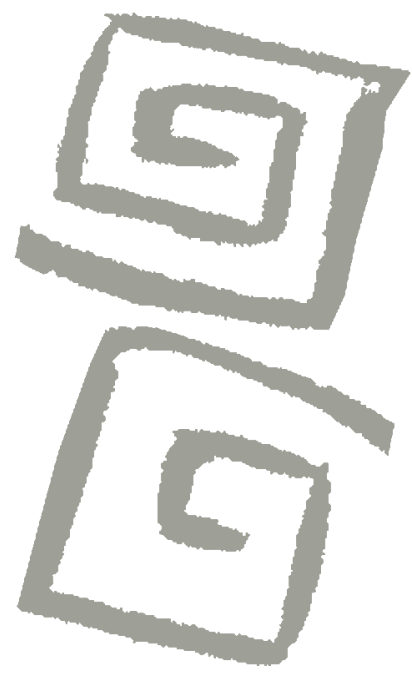

\title{
Espiritualidad y salud: problemas de salud durante el embarazo y el puerperio y sus consecuencias en las trayectorias de mujeres y niños (Salta, Argentina)
}

\author{
Spirituality and health: problems during pregnancy \\ and postpartum and their consequences in women and \\ children's life trajectories (Salta, Argentina)
}

\author{
Carolina Remorini', María Laura Palermo², Lucía Schvartzman ${ }^{3}$
}

'Doctora en Ciencias Naturales. Profesora e investigadora adjunta, Laboratorio de Investigaciones en Etnografía Aplicada, Universidad Nacional de La Plata Consejo Nacional de Investigaciones Científicas y Técnicas. La Plata, Argentina. $\triangle$ iD

2Licenciada en Antropología. Becaria Doctoral, Laboratorio de Investigaciones en Etnografía Aplicada, Universidad Nacional de La Plata, Consejo Nacional de Investigaciones Científicas y Técnicas. La Plata, Argentina. $\square$ (iD)

${ }^{3}$ Estudiante de Antropología. Docente auxiliar, Laboratorio de Investigaciones en Etnografía Aplicada, Universidad Nacional de La Plata. La Plata, Argentina. $\Delta$ iD
RESUMEN Este trabajo aborda la relación entre salud y espiritualidad a través del análisis de la narrativa de padecimientos que ocurren durante el embarazo y el puerperio y afectan a mujeres y niños en comunidades rurales de Molinos (Valles Calchaquíes, Salta). Se basa en una investigación desarrollada entre 2009 y 2017, en el marco de la cual se realizaron 33 entrevistas semiestructuradas a mujeres cuidadoras de niños menores de seis años, y en la que se focalizó en el modo en que las entrevistadas presentaban las secuencias de eventos que rodeaban la emergencia de problemas de salud propios y de sus hijos, en las que se conjugan aspectos orgánicos, emocionales y espirituales tanto en su etiología como en su terapéutica. Tomamos como ejemplos de análisis el susto y sus variantes, la recaída y la matriz. Los sentidos otorgados a estos padecimientos ponen de relieve la dimensión espiritual que justifica la apelación a recursos médicos tradicionales. Nuestro enfoque busca trascender la perspectiva taxonómica y esencialista para dar cuenta de los aspectos procesuales involucrados en la experiencia de enfermar.

PALABRAS CLAVES Etnografía; Salud Materno-Infantil; Espiritualidad; Argentina.

ABSTRACT This article addresses the relationship between health and spirituality through the analysis of narratives of illnesses that occur during pregnancy and postpartum and that affect women and children in rural communities of Molinos (Calchaquí Valleys, Salta). It is based on research carried out from 2009-2017, in which 33 semi-structured interviews were conducted with women caring for children under six years of age. We focused on the way in which the interviewed women presented the sequence of events surrounding the emergence of illnesses affecting their own health and that of their children, in which organic, emotional and spiritual aspects interact in both the etiology of the illness and its treatment. We analyze as an example susto [fright] and its variations, as well as recaida [relapse] and matriz [womb]. The meanings attributed to these illnesses stress the spiritual dimension involved that justifies the use of traditional medicine resources. Our approach seeks to go beyond a taxonomic and essentialist perspective to focus on aspects involved in the process of the experience of illness.

KEY WORDS Ethnography; Maternal and Child Health; Spirituality; Argentina. 


\section{INTRODUCCIÓN}

La relación entre salud y espiritualidad es objeto de reflexión y debate en las sociedades contemporáneas, en la búsqueda de alternativas para el logro y mantenimiento del bienestar en momentos críticos de la trayectoria vital. Nuevas y viejas experiencias en torno a la espiritualidad confluyen en escenarios multiculturales y se constituyen en alternativas para el cuidado de la salud. Así, emerge y se resignifica la necesidad de un equilibrio cuerpo/mente/espíritu para afrontar padecimientos y crisis, particularmente, en etapas de la vida en las que los sujetos se perciben vulnerables. Ante el cada vez más diverso conjunto de prácticas vinculadas a la espiritualidad en las comunidades cosmopolitas que combinan tradiciones, saberes y valores heterogéneos, las instituciones biomédicas comienzan a mostrarse más receptivas, al punto de incorporar algunas de estas prácticas de manera complementaria a sus tratamientos, para responder a demandas y necesidades de los pacientes ${ }^{(1)}$. No obstante, resulta claro que este proceso no ocurre sin conflictos, dando lugar a numerosas discusiones sobre la legitimidad terapéutica de dichas prácticas. Si bien la biomedicina reconoce la incidencia de factores no orgánicos en el estado de salud -de hecho la definición de la Organización Mundial de la Salud ${ }^{(2)}$ repetida incansable y acríticamente refiere al completo bienestar bio-psico-social- el abordaje biomédico de estos aspectos reconoce actitudes que van desde la sanción a la derivación a otros terapeutas o especialistas, pasando por formas de aceptación pasiva o apropiación ${ }^{(3)}$. En general, el paciente y su entorno son quienes realizan la articulación entre diagnósticos y terapias, lo que se materializa en trayectorias que combinan diferentes prácticas efectivas y aceptables -desde el punto de vista del paciente y su entorno sociocultural- para la recuperación de su salud y el mantenimiento del bienestar.

Este escenario de pluralismo terapéutico en las sociedades cosmopolitas modernas, en el que la relación entre salud y espiritualidad se ha resignificado, se percibe como un signo de época ${ }^{(1)}$. Sin embargo, la constatación de tales articulaciones terapéuticas no resulta novedosa desde la perspectiva antropológica. Si bien se supone la hegemonía de la biomedicina en el cuidado y atención de la salud -a diferencia de lo que se asume para las comunidades Ilamadas "tradicionales"- las investigaciones en antropología médica han mostrado los límites de estas asunciones. Autores como Taussig ${ }^{(4)}$, Kleinman ${ }^{(5)}$, Douglas ${ }^{(6)}$ y $\operatorname{Good}^{(7)}$ han sido, entre otros, quienes aportaron a visibilizar la pluralidad de saberes, valores y prácticas que los pacientes conjugan en las decisiones sobre su salud, poniendo de relieve que el uso de múltiples terapias de manera complementaria o alternativa es más una regla que una excepción ${ }^{(8,9)}$. $\operatorname{Good}^{(7)}$ ha identificado así la existencia de un "sistema etnomédico" producto de la conjunción entre la biomedicina, la medicina practicada por curanderos, el autotratamiento y las medicinas religiosas y alternativas ${ }^{(8)}$.

Aunque estas prácticas no son privativas de las comunidades "tradicionales", los estudios sobre complementariedad terapéutica se enfocaron inicialmente en contextos rurales -comunidades campesinas e indígenasy analizaron las tensiones derivadas de los desencuentros entre la medicina científica y la tradicional, a partir de la expansión de los programas y servicios de salud biomédicos a regiones diversas del Tercer Mundo. Los estudios sobre etnomedicina ${ }^{(10,11,12)}$ se originaron en contextos etnográficos clásicos, es decir, fuera de occidente, como derivados de los estudios sobre religión "primitiva" y estudiaban "aquellas creencias y prácticas relativas a la enfermedad", producto del "desarrollo cultural indígena"(13).

Estos trabajos abordaban las clasificaciones de enfermedades y terapias tradicionales derivadas de la "cosmovisión"(10) de manera independiente de aquellas derivadas del contacto y colonización europea. Rivers fue uno de los primeros en establecer que la magia, la religión y la medicina estaban íntimamente entrelazadas en estas comunidades indígenas, y no era posible estudiar una sin 
las otras. A ello se oponía el estudio de las prácticas médicas en sociedades occidentales, visión criticada por Foster ${ }^{(14)}$.

Esta mirada sobre la relación entre medicina y religión en comunidades étnicamente distintivas, perduró en los estudios etnomédicos en Latinoamérica, y Argentina no fue una excepción. Los estudios se concentraron, principalmente, en sociedades indígenas y criollas rurales, abordando las creencias y prácticas religiosas involucradas en la etiología y terapia de la enfermedad.

En el caso de las comunidades del noroeste argentino, las creencias y prácticas médicas tradicionales articulan las dimensiones espirituales y orgánicas en el diagnóstico y curación de numerosas dolencias ${ }^{(15,16,17,18,19)}$. En este marco se inscribe la investigación desarrollada durante las últimas décadas por investigadores del equipo al que pertenecen las autoras de este trabajo en los Valles Calchaquíes salteños. Algunas de las dolencias del corpus médico local han sido clasificadas como "enfermedades del espíritu"(15) ya que su manifestación compromete el componente espiritual de la persona. Tanto a nivel de las creencias como de las prácticas, que combinan el tratamiento empírico con rituales mágico-religiosos, se observa una yuxtaposición de elementos indígenas y del culto cristiano $^{(15,20)}$.

En el marco de investigaciones actuales que dieron lugar a numerosas publicaciones ${ }^{(20,21,22,23,24,25,26,27,28,29,30,31,32)}$ se ha avanzado en el análisis de narrativas que dan cuenta de la imbricación de aspectos psicoemocionales y orgánicos en la etiología de diversos padecimientos. Ello justifica enfocar en las experiencias de quienes las padecen y en cómo se articulan síntomas y tratamientos en secuencias complejas que conjugan diversos aspectos de la trayectoria vital de los sujetos afectados. Es así que síntomas y dolencias cobran nuevos sentidos al ser reinterpretados a la luz de experiencias vitales concretas.

En este artículo caracterizamos percepciones y experiencias de mujeres de diferentes generaciones en torno a un conjunto de padecimientos que experimentan durante el embarazo y puerperio, que impactan en etapas posteriores de su trayectoria vital y/o la de sus hijos. Estos son el susto y sus variantes (aikado, quedao), la recaída y la matriz. En las páginas que siguen analizamos el modo en que se describen estos padecimientos y las categorías que aluden a ellos y sus síntomas, así como los diversos sentidos que se les asignan en los relatos de mujeres de diferentes generaciones del departamento de Molinos (Salta, Argentina). Este análisis se organiza en función de tres ejes íntimamente vinculados: 1) las causas raíz (no inmediatas) de padecimientos sufridos por ellas y/o sus hijos que se manifiestan en la etapa pre y postnatal, pero reconocen antecedentes en momentos previos de sus trayectorias vitales; 2) los efectos de tales padecimientos en el estado emocional, comportamiento habitual y el desarrollo de competencias psicofísicas y sociales a corto y largo plazo; 3) la indisociabilidad de los componentes orgánicos, espirituales y sociales en su etiología, desarrollo y terapia.

\section{Las comunidades vallistas}

El departamento de Molinos, localizado en los Valles Calchaquíes septentrionales en la provincia de Salta, tiene una población total de 5.565 habitantes $^{(33)}$. Esta región, ubicada al noroeste de Argentina, registra un poblamiento continuo a lo largo de, al menos, dos mil quinientos años. La población actual representa culturalmente una superposición y fusión parcial de elementos indígenas e hispánicos ${ }^{(34)}$. Investigaciones previas ponen de relieve la diversidad ecológica que exhibe la región, lo que impacta en las estrategias de vida de sus pobladores. En términos de esta diferenciación ecológica, se distingue entre el pueblo de Molinos, cabecera del departamento y las áreas rurales denominadas "fincas". Ello se corresponde asimismo con la distinción local entre el bajo y el alto o los cerros, respectivamente ${ }^{(27)}$.

El pueblo es sede de actividades administrativas, políticas, comerciales y de servicios. Las familias residentes en las fincas practican fundamentalmente tareas agrícologanaderas, cuya producción conjuga la 
permanencia de utilización de mano de obra (arriendo, mediería y pastaje) con un tipo de explotación agrícolo-industrial, en especial vitivinícola. El avance de esta modalidad productiva conlleva una notable disminución de la población en las fincas y un cambio en las prácticas de subsistencia de las unidades domésticas, junto con la relocalización de la población desplazada en el pueblo u otros lugares dentro y fuera de la región ${ }^{(35)}$. En las unidades domésticas de las fincas prevalece la división del trabajo por género: los hombres adultos, jóvenes y niños se dedican al trabajo agrícola en parcelas propias o arriendos, trabajan de manera asalariada en las fincas en diversas tareas e intervienen en las actividades vinculadas al manejo del ganado vacuno, principalmente; mientras que el cuidado de la "hacienda" (ovejas, cabras, chivos, Ilamas) es una responsabilidad mayoritariamente asumida por las mujeres adultas. Estas, junto a niños de ambos géneros se ocupan diariamente del ganado, y de las labores vinculadas a la elaboración de comida, limpieza, lavado de ropa, recolección de leña y agua y trabajo textil. Algunas también participan de las actividades agrícolas en ausencia de los hombres. En el pueblo y orillas, hombres y mujeres tienen menor dedicación a las actividades "tradicionales", ya que pocas familias poseen espacios para el cultivo y cría de ganado. En su lugar, adquieren relevancia el comercio, el trabajo asalariado y nuevas oportunidades de formación y práctica profesional. Aquí las mujeres permanecen vinculadas a las labores en el hogar y el cuidado de los hijos, y las pocas que trabajan fuera lo hacen mayoritariamente en empleos informales.

Tanto en el pueblo como en las fincas, un elevado número de unidades domésticas es de tipo extenso y matrifocal, lo que responde a múltiples factores tales como migración asociada a la búsqueda de trabajo y educación de nivel terciario o universitario, abandono del cónyuge o desconocimiento de la descendencia, y/o elección de no conformar una pareja estable. En el pueblo, al momento de la investigación, el promedio de hijos era de cuatro, mientras que en las orillas y fincas se ubicaba entre siete y ocho, lo que da como resultado que el cuidado de los niños pequeños se distribuya entre la madre, alguna otra mujer joven (generalmente una hermana soltera), alguna mujer anciana y sus hijos mayores. Tradicionalmente, Molinos se caracterizó por la abundancia significativa de unidades domésticas matrifocales con generaciones alternas ${ }^{(21,31)}$. Considerando la ausencia temporaria o definitiva de individuos jóvenes y adultos, los más viejos -abuelos sanguíneos o putativos- suelen asumir la crianza y cuidado de la generación de los nietos. Este modo de organización resulta recurrente y se mantiene a través del tiempo.

En este contexto, las mujeres ancianas son referentes para la crianza y el cuidado de la salud en momentos críticos del curso vital; su experiencia es fuente de hipótesis para la toma de decisiones sobre alternativas de cuidado de la salud en un escenario actual de mayor disponibilidad de recursos, saberes y actores con diferentes niveles de legitimación.

Entre el pueblo y las fincas existen diferencias en la disponibilidad y accesibilidad de servicios biomédicos. En el pueblo se encuentra el Hospital Provincial Dr. Juan A. Fernández, mientras que en las fincas encontramos seis puestos sanitarios que cuentan con un enfermero y/o un agente sanitario. El hospital atiende patologías de primer nivel de complejidad y partos y deriva las patologías más severas y cesáreas a centros de mayor complejidad. Los profesionales médicos realizan visitas semanales programadas a cada una de las fincas. Estos son los únicos servicios biomédicos disponibles para los pobladores, los que en su mayoría no poseen obra social ni cobertura privada. No obstante la mayor accesibilidad a recursos biomédicos en los últimos años, continúa siendo problemática la resolución de emergencias, complicaciones durante el parto y la atención de cesáreas por falta de personal especializado.

La mayor utilización de servicios biomédicos se complementa actualmente con actores, saberes y recursos de la "medicina tradicional". Numerosos padecimientos continúan siendo tratados en las unidades domésticas o 
mediante el recurso a los "médicos campesinos", en los casos de enfermedades que no son conocidas ni tratadas por la biomedicina, para complementar tratamientos médicos o en diferentes instancias del embarazo, parto y puerperio ${ }^{(20,21,31)}$.

\section{ESTRATEGIAS TEÓRICO- METODOLÓGICAS}

Nuestra investigación reconoce sus antecedentes en estudios iniciados en la década de 1970 en el departamento de Molinos ${ }^{(34,36,37,38,39,40,41)}$. Gran parte de este primer corpus documental describe enfermedades culturalmente específicas que afectan a los individuos en diferentes etapas de su vida, así como las referencias a creencias y prácticas en torno a su etiología, sintomatología y terapéutica. Como resultado, emergen problemáticas que justifican la necesidad de atender a la variabilidad de creencias y prácticas en torno al cuidado de la salud, incorporando la metodología de casos en el abordaje de las estrategias frente a la enfermedad en su articulación con otros dominios de la vida colectiva, trascendiendo los discursos expertos ${ }^{(34)}$.

Las investigaciones se retoman en el año 2009, orientadas al estudio de las prácticas de crianza y cuidado de la salud de los niños, a través de la narrativa de las cuidadoras y de la observación de sus rutinas domésticas e itinerarios cotidianos, con el objetivo de identificar, caracterizar y evaluar los factores que configuran el nicho de desarrollo ${ }^{(42)}$ y su incidencia en las trayectorias de salud de los niños desde una perspectiva ecológica(43,44). A partir de allí, se abre una línea de indagación centrada en el estudio de casos e itinerarios vinculados al cuidado y atención de la salud materna e infantil en la etapa perinatal. En este marco, nos interesa la descripción y análisis comparativo de secuencias terapéuticas que involucran, por ejemplo, episodios de susto, y otras categorías vernáculas de enfermedad, identificando actores, vínculos y recursos que se seleccionan y articulan en su desarrollo. Estas secuencias nos interesan en tanto conectan las trayectorias de salud de las madres y de sus hijos, y ponen de manifiesto las expectativas y valores en torno al crecimiento y desarrollo de los niños ${ }^{(32)}$.

Desde el punto de vista metodológico estas investigaciones siguen un diseño de tipo cualitativo y se basan en la utilización complementaria de entrevistas semiestructuradas, observaciones (sistemática, spot y participante) y registro audiovisual. La información discursiva en la que se basa este artículo surge de entrevistas realizadas a 33 mujeres de diferentes edades (18 a 70 años) cuidadoras de niños menores de 6 años, algunas de las cuales han sido entrevistadas en más de una oportunidad entre 2009 y 2017. Ellas fueron seleccionadas en función de integrar unidades domésticas de diferente composición, localización y tipo de subsistencia, así como por su acceso diferencial a instituciones educativas y sanitarias. Cada entrevista tuvo una duración de una hora y media en promedio.

Del total hemos seleccionado 12 relatos que dan cuenta de las secuencias de interés para el análisis e ilustran la diversidad de trayectorias vitales del conjunto de las entrevistadas. Para cada uno identificamos categorías utilizadas para referir a padecimientos sufridos durante el embarazo, parto y puerperio, así como expresiones empleadas para describir los síntomas y la secuencia de diagnósticos y tratamientos aplicados. Asimismo, delimitamos los eventos que precedieron y sucedieron a los episodios referidos. Analizamos comparativamente los relatos a fin de reconocer y caracterizar recurrencias y diferencias mediante Nvivo 10. Este software permite formalizar, codificar y sistematizar los datos cualitativos e identificar categorías para la descripción del campo conceptual y sus relaciones semánticas.

Esta investigación ha sido aprobada desde el punto de vista ético por el Comité de Bioética de la Facultad de Artes y Ciencias (Universidad Católica de Salta). Las entrevistadas prestaron su consentimiento libre e informado de acuerdo a lo establecido en la Ley 25326 y los datos personales han sido protegidos mediante la utilización de iniciales. 


\section{RESULTADOS}

\section{Trayectorias vitales y experiencias de maternidad}

En esta sección describimos la manera según la cual las entrevistadas articulan en su narrativa diferentes aspectos de sus trayectorias vitales y experiencias de maternidad con la secuencia de eventos que rodean la emergencia y desarrollo de dolencias incluidas en el ámbito de la medicina tradicional de la región andina: el susto y sus variantes (aikado, quedao), la recaída y la matriz.

Según la caracterización más general del susto, este origina un desequilibrio cuerpoespíritu, específicamente la pérdida temporal del espíritu, presentando síntomas emocionales y físicos a la vez ${ }^{(20)}$. Las situaciones pasibles de provocar susto refieren a entornos y circunstancias inesperadas o contrarias a las expectativas del individuo (consecuencias de fenómenos naturales, haber presenciado accidentes o fallecimientos, caídas, ataques de animales, visiones o contacto con entidades sobrenaturales).

A los fines de este artículo nos enfocamos en las referencias al susto que surgen de relatos sobre eventos disruptivos durante el embarazo y puerperio, ocasionando diversos padecimientos $\mathrm{y} / \mathrm{o}$ problemas de desarrollo en las mujeres o sus hijos. Estos eventos traumáticos pueden tener lugar durante las actividades cotidianas, ser consecuencia de la violación de tabúes (contacto con los muertos) o de transitar espacios considerados "peligrosos" (como el bajo, el río o el cementerio). En estos espacios, alejados del emplazamiento de las viviendas, las personas pueden tener encuentros con seres sobrenaturales. En el caso del río, la fuerza, potencia y capacidad de acarreo, pueden ser consideradas negativas, ya que el agua puede arrastrar animales y personas, y los eventos de desborde de los ríos provocan intranquilidad y temor ${ }^{(35,45)}$.

...este [su hijo mayor] jsiempre se ha asustado! [...] no crecía y eso nos Ilamaba la atención [...] le hemos llevado a curar a Doña I. [médica campesina] y ella me ha dicho que era aiqueado, así le llaman, que es cuando una está embarazada y va a un velorio y se asusta o se aflige y entonces el chico ya desde la panza viene mal, ya no crece [...] dicen que es porque te afligís ahí en el velorio o porque te asustás con el muerto. (MT, 44 años, Molinos)

[cuando estás embarazada] dicen que te asustas vos y se asusta el bebé y después el bebé de la panza ya sale asustado [...] Por ahí no puede estar tranquilo, no puede dormir. (IL, 25 años, Molinos)

Lo más feo es con el río, dicen que se asustan los bebés. Porque dicen que es jodido con el río que le lleva el espíritu [del niño], [hay que] Ilamarlo porque ya se lo lleva el río [al espíritu]. (PR, 41 años, Tomuco)

$\mathrm{Si}$ es curado de manera inmediata, el susto no tiene efectos severos a largo plazo, pero posponer su tratamiento o interrumpirlo resulta en trastornos más severos, que se manifiestan a lo largo de la trayectoria vital. En este sentido, puede derivar en otros problemas de salud de variable intensidad y gravedad.

Las mujeres durante el embarazo y los niños pequeños ("guaguas") son particularmente susceptibles a sufrir susto. Una variante denominada aique refiere a episodios que sufre la mujer gestante y que ocasionan en el niño una elevada susceptibilidad a enfermar y sufrir trastornos de desarrollo (problemas motrices, falta de tonicidad muscular, sordera, mudez, ceguera). Se considera que las guaguas son "tiernitas" (frágiles) y que se "asustan de cualquier cosa". Ello se debe a que su espíritu se halla débilmente unido al cuerpo y puede separarse o huir ${ }^{(46)}$. También el espíritu puede huir debido a eventos que ocurren más allá de la etapa gestacional, por ejemplo, cuando los niños se sienten atraídos por alguna entidad sobrenatural que, en general, se halla en lugares aislados o inhabitados. El signo principal de esta situación es que los niños "no quieren regresar" o "siempre quieren ir y no volver". 
"Nacer aicado" o un "susto mal curado" tiene consecuencias en su conducta, estado emocional y sociabilidad. En los relatos, estos efectos se expresan como: ser malito, Ilorón, renegón, pegote, estar molesto, estar triste, ser desesperado, loquito, "todo el tiempo quiere llamar la atención".

...los míos [sus hijos] todos son asustados [...] son malos, se ponen a llorar, por ejemplo, quiere ofrecer algo, él es malo, se enoja, no quiere recibir, se pone inquieto, como que no hacen caso, como que no estuvieran escuchando [...] [tienen] bajo peso también, pero yo más les veo renegones. (DF, 52 años, Molinos)

[su hijo] aumentaba de peso, pero poquito, era pícaro, terrible, su cuerpito bien flaquito. (MT, 44 años, Molinos)

Cuando estos problemas persisten en la niñez e incluso adultez, significa que la curación no ha sido efectiva y oportuna, y se dice que el individuo es quedao ${ }^{(15,20)}$. Esta expresión alude a un retraso madurativo ("se ha quedao"; "empieza a atrasarse la persona") que se manifiesta en la conducta y reduce las posibilidades de integración de los niños a actividades de valor para sus trayectorias futuras.

...era más flojito [que sus hermanos], demoró mucho para caminar [...] yo ya lo veía distinto, que no escuchaba, entonces me preocupaba mucho y casi siempre iba al hospital y me dijeron que era sordito [...] desde que se enfermó casi todos los médicos campesinos que hay acá le llevaba, la mayoría me ha dicho que él ya es para que sea un chiquito mudo... (PC, 30 años, Churkal)

...porque esta [niña] ya tiene seis años y ella vive así, asustada porque cuando ha sido ese incendio, ella quedó como asustada ya, y yo le he dicho a su mamá, "hacela curar, hacela llamar" [pero] no la ha hecho llamar, y no se ha compuesto bien. (DF, 52 años, Molinos) ...todos los que lo vieron [a su hijo] dijeron que está muy asustado desde el vientre. $Y$ es verdad, yo me asusté con él dentro de la panza [...] así que le llevé a dos, tres señores y los tres me dijeron lo mismo, porque él está fuerte asustado y le dura. Él era así como desesperado, loquito [...] todo el tiempo quiere llamar la atención [...] cuando ellos se asustan es como que quedan para el lado del mal [...] Una señora me dijo que eso se les sube a la cabeza, que es como que quedan así locos, trastornados. (NM, 34 años, Molinos)

...vos no le hiciste curar de chico entonces cuando es más grande como que tienen una ira, vos no sabes qué les pasa, por qué tienen temperamento. (IL, 25 años, Molinos)

...por ahí hay muchas veces que cuando ya van a la escuela, no prestan atención bien al estudio [...] ahí están asustados, están pensando en otra cosa menos en el estudio, están en otro mundo, digamos. (DF, 52 años, Molinos)

...una vez un primo nuestro [...] sabía ir a jugar mucho en la playa [...] una noche llegó descalzo, con fiebre, temblando y sudando así de miedo [...] después ya estaba mal, estaba así como loco [...] le ha hecho curar mi tía pero él ha quedado así intranquilo, trabaja en un lado, ya deja, que va a otro lado. $Y$ así es, no se estabiliza nunca, es así como intranquilo, no tiene una tranquilidad o estar seguro. (NM, 34 años, Molinos)

Según nuestros hallazgos el aique se vincula a experiencias y estados emocionales negativos durante el embarazo, que se describen como estar angustiada, Ilorar mucho, estar triste, tener miedo a todo, ser nerviosa.

...los médicos campesinos siempre me decían que yo sufrí mucho cuando quedé embarazada, es verdad, siempre me ponía a llorar porque tenía miedo 
de todo, siempre me la pasaba Ilorando, como angustiada y me asustaba [...] cuando estaba quedándome embarazada me fui a trabajar con mi papá y había pasado una víbora por ahí, por mi cuerpo [...] ahí fue cuando más me asusté, de ahí ya no era como era antes, ya tenía miedo a todo, ya por cualquier cosa me ponía a llorar. (PC, 30 años, Churkal)

...ni se me cruzó en la cabeza que podía estar embarazada y claro, después yo no lo asimilaba. Y encima también estaba un poco mal porque yo ya venía muy distanciada digamos del papá de $X$ [su hijo]. $Y$ después para navidad la pase re mal, porque me hizo re mal, tenía un dolor acá, el único síntoma era en el estómago [...] Tenía miedo, tenía terror. No sabía cómo iba a afrontar eso y encima estaba sola [...] Él [su hijo] debe ser así como nervioso [...] porque yo la pasé así [...] La cosa que después recién los últimos meses ya me tranquilicé, había aceptado que me fue mal y se me había juntado todo: la separación del papá de $X$, mi frustración por la universidad, no sé, como fracasos [...] y resulta que me pego un susto en el embarazo que rompo bolsa [...] yo me asusté porque estábamos haciendo empanadas [...], entonces yo vi que se estaba quemando una empanada y pedí ayuda [...] Y va mi sobrinito, apaga la hornalla y se [me] resbala la sartén y le quema todas las manos con el aceite caliente, horrible, yo me re asusté [...] faltaban dos semanas todavía, un poquito más [para el parto] la cosa es que rompo bolsa, después me tocó un parto seco, no tenía dilatación, no nacía X. (NM, 34 años, Molinos)

Así, la caracterización de las circunstancias que provocan susto guardan correspondencia -al menos parcialmente- con otros estados descritos para comunidades andinas, como la pena y los nervios ${ }^{(34,47,48)}$, específicamente cuando el susto se vincula a situaciones adversas que predisponen a un estado emocional negativo, como la soledad, la incertidumbre, la falta de apoyo social, la carga de responsabilidad, las expectativas en torno a proyectos de vida o a los hijos. Esta condición de susceptibilidad individual se combina en los relatos con sucesos inesperados o repentinos que desencadenan el susto.

De lo expuesto hasta aquí se desprende que en la reconstrucción de la secuencia de eventos que desencadenan problemas que preocupan a las madres, la causa última de estos es atribuida tanto a un susto mal curado, un susto en la niñez o a haber nacido aicado. Así, la posibilidad de quedar asustada/o puede resultar de la no resolución o acumulación de experiencias negativas junto con la ineficacia de la terapia ${ }^{(47)}$.

...yo sentía como que estaba depresiva [...] De hecho por eso también él [su hijo] debe ser así como nervioso [...] Yo le hice curar una o dos veces, en realidad dicen que se curan con tres veces y entonces yo no hice la tercera...no le curé como me pidieron porque él no quería tomar algunos remeditos [...] o sahumarle y él no se dejaba, después tenía que limpiarle con ruda porque [el médico campesino] me decía que era muy nervioso, muy asustado" (NM, 34 años, Molinos)

De este modo, el susto suele ser la causa última de un conjunto de problemas que se prolongan en el tiempo, y se articulan en secuencias no lineales y complejas en las que diversos tratamientos y diagnósticos se eligen, descartan, confirman y/o redefinen. Las consecuencias a nivel físico/orgánico se dan simultáneamente a las de orden espiritual. Por ejemplo, si el cuerpo se abre a consecuencia del susto -provocando la pérdida del espíritu- ello puede combinarse con otros síntomas (diarrea, vómitos) dando lugar a un cuadro complejo que requiere de una combinatoria de acciones terapéuticas.

Los problemas del espíritu solo pueden ser reconocidos y tratados por aquellas personas cuya condición espiritual sea incuestionable, debido a su devoción y fortaleza. Se subraya que estas personas deben ser baqueanas (experimentadas) en virtud de 
los riesgos que ello supone, ya que puede incluir encuentros y disputas con entidades sobrenaturales.

La principal acción terapéutica es el llamado del espíritu por parte de miembros de la unidad doméstica (fundamentalmente ancianas y ancianos) de manera inmediata tras el evento que provocó el susto. De persistir los síntomas se recurre a médicos campesinos $^{(37)}$, quienes además pueden administrar remedios de yuyos (combinatorias de plantas medicinales) por vía oral, realizar limpias, refregadas (masajes o fricciones) y manteadas cuando es necesario cerrar el cuerpo.

[cuando está asustado] abre los brazos, así grande, y se pone duro. Mi mami nomás curaba, ella decía vos te has asustado, te tocaba acá y decía que se te había abierto el pecho. Ella con una manta te movía, porque cuando te asustas, te agarra vómito, de todo un poco. $Y$ así ella me curaba, con la manta y me envolvía el pecho. (YC, 25 años, Molinos)

...mi mamá me lo llama [a su hijo], agarra agua bendita, le da de tomar un poquito, y le dice asi 'veni $Z$, veni hijito, no te quedés, no tengas miedo, veni' y después le da de tomar más agua, le hace crucitas [cruces] por acá [en la coronilla] reza y luego ya está, no sé si será creencia o qué pero se compone. (MT, 44 años, Molinos)

...también decía mi mamá "hay que sahumarles", y ella les hacía un remedito, junta todos los yuyitos [...] plumitas de todos los pajaritos del campo, entonces con eso hay que sahumarle [...] ella los pone así a los chiquitos arriba [del humo]. (SR, 43 años, Molinos)

La terapia da cuenta del sincretismo con creencias y prácticas provenientes de la religión católica, tales como la invocación a santos y vírgenes, las plegarias, elementos como agua bendita, incienso, velas e imágenes, la alusión a los días martes y viernes y la recurrencia de los números 3 y 9 como plazos y frecuencias ideales para la aplicación y/o duración de los tratamientos. La apelación a la biomedicina se limita al tratamiento de algunos síntomas orgánicos, de manera simultánea a la atención por los médicos campesinos ${ }^{(20)}$. En este sentido, nuestros datos nos permiten afirmar que la eficacia de los recursos y procedimientos terapéuticos derivan de su combinatoria y no solo de las propiedades de cada uno ${ }^{(15)}$.

Las consideraciones realizadas en torno al susto pueden extenderse al análisis de otras categorías nosológicas locales como la recaída, que refiere a un conjunto de síntomas y malestares que pueden producirse en los días posteriores al parto debido a la exposición de la puérpera a situaciones que suponen un desbalance frío/calor ${ }^{(49)}$, como contacto con el agua, exposición al fuego o al viento (aire). Para evitarla deben cumplirse un conjunto de prescripciones que implican una restricción de los espacios y actividades cotidianas. La reclusión es la medida preventiva central, e implica que la puérpera debe permanecer en un espacio cerrado de la vivienda, sin exposición a cambios de temperatura, realizar reposo y seguir una dieta de comidas livianas. Esto supone que otras mujeres de la unidad doméstica se hagan cargo de las responsabilidades de las cuales la puérpera queda relevada. Asimismo, las mujeres luego del parto deben ser fajadas para evitar que sus cuerpos se $a b r a n^{(39)}$, ya que el cuerpo abierto está expuesto a la acción patogénica de entidades naturales y sobrenaturales ${ }^{(46,50,51)}$. Al igual que con el susto, la posibilidad de que el cuerpo se abra o se enfríe se relaciona con diversas circunstancias ordinarias y extraordinarias, tanto en el caso de los niños como de las mujeres en diferentes momentos de su ciclo reproductivo. La terapia incluye, además, la administración de infusiones a base de plantas medicinales y menos frecuentemente sahumado y fricciones (refregadas).

...quince días me han tenido en cama [...] dicen que tenés que estar en cama porque es peligroso las recaídas, dice 
que [hace mal] el sol, el agua, todo eso, ¿ve? ella [su mamá, hace las cosas de la casa] porque ella está sana, ella hace todo lo que se pueda [...] por ahí me tienen que fajar o va a quedar desarmado el cuerpo [...] si yo tenía [un parto] en el hospital a los dos días ya me hacían levantar, ya tenía que lavar todo, a los tres días ya salía al sol [...] ya a partir de cinco días estaba aquí [en su casa], ya cocinaba, ya lavaba, ya salía al sol. (EG, 41 años, Gualfín)

La narrativa recopilada da cuenta de discrepancias en el grado de adhesión a estas pautas ("las creencias de las mamis") entre mujeres de diferentes generaciones.

...antes te cuidaban mucho después de parto... en ese tiempo me acuerdo que la mamá me tenía en cama, que no entre en una recaída [...] según sus creencias, de antes, que si vos te levantás te da el aire y te puede dar dolor de cabeza, [hay que hacer] reposo en la habitación, que no te de mucho aire, viento, frío [...] Y no tocar agua, con alcohol nos limpiaban las mamis [...] de eso tenía yo miedo, de la recaída. "Porque viste que algunas hasta se pueden morir", decía la mami. (MT, 44 años, Molinos)

...después que los tuve [a sus hijos] me he quedado con un poco de dolor de panza y ella siempre me daba un tecito de orégano... que era para limpiar todo el resto de sangre que queda [...] nos tenía más o menos quince días sin bañarnos... no quería que nosotras salgamos, teníamos que estar encerradas quince días, agua fría o agua hirviendo no tenías que usar, nos cuidábamos de no estar cerca del fuego y no levantarse... después del mes recién te arrimabas al fuego... porque dice que es de recaidoso, ya venía dolor, te dolían los pechos y la cabeza. (DF, 52 años, Molinos)

...mi mamá dice que no tenemos que arrimarnos al fuego y después no comer las comidas pesadas porque le caen mal al bebé, por la leche... no acercarse al fuego, no hacer horneadas... decía siete días hay que cuidarse [...] Sufrí un montón, siempre me persigue el dolor de cabeza, mi mamá dice [que] porque a veces es que no te cuidas del parto... recaída ella dice... Y después nos sabía decir que no nos desabriguemos mucho porque se te enfría la espalda y después ya no tenés leche para el bebé. (CC, 23 años, Santa Rosa)

Al igual que con los sustos no curados, puede ocurrir que los síntomas se prolonguen en el tiempo y se articulen con otros nuevos, dando origen a padecimientos más complejos. Así, ser recaidosa significa que la mujer ha sufrido en más de una oportunidad una recaída que no fue tratada efectivamente, colocándola en una situación de mayor susceptibilidad. Ello explica la recurrencia de síntomas tanto físicos como emocionales que impiden a las mujeres llevar a cabo sus actividades cotidianas. En este sentido, en la explicación de síntomas o dolencias actuales, tiene un rol central la apelación a padecimientos que afectan al individuo en momentos claves de su trayectoria vital (como el embarazo y el puerperio) y que no han sido debidamente tratados.

...me han dicho eran recaídas y esas cosas que me pasaban, yo me he hecho curar con médico campesino y me dice que son recaídas los dolores de hueso, dolores de mano, que yo no puedo lavar, dolor de cabeza, mucho, transpiro [el médico campesino] dice que eso son recaídas [...] y también son artrosis [...] porque no me cuidaba en esos días de parto... quizás que ahí me habré cuidado poco, porque ya mis hijos eran grandes. Antes yo me he recaidado mucho, y he tenido el tercer, cuarto, quinto hijo, por ahí me he recaidado grave. $Y$ bueno, el primero o el segundo quizás también, porque yo no sentía nada, entonces ya [me] levantaba [...] y después sentía los 
dolores de cabeza [...] así me han dicho los médicos campesinos a mí, usted tiene muchas recaidas de antes, que usted y su hijo no se han cuidado nada del fuego, del agua, del sol, eso son lo que ahora están apareciéndome [...] [ahora] yo salgo mucho al sol y hay dolor de cabeza, dolor de cuerpo así, dolor de hueso... te quita la fuerza. (EG, 41 años, Gualfín)

En el caso de esta entrevistada, asocia los episodios reiterados de recaida a actividades que realizó durante el embarazo y puerperio, desatendiendo las recomendaciones que rigen para estas etapas. En su relato aparecen reiteradamente expresiones que remiten a su responsabilidad casi exclusiva en el sostén de sus hijos y su falta de apoyo ("estaba siempre sola", "tenía que hacer mis cosas", "¿quién me va a ayudar?") debiendo continuar con las tareas domésticas y la actividad artesanal para la venta:

...mucho sabía trabajar con esta lana, porque antes de tener un hijo una no se cuida nada, después te amortigua, te duele, porque la lana de llama es recaidosa, tiene mucho aire, airosa, por eso no tenemos que trabajar con la lana hasta quince días, un mes [después del parto]. (EG, 41 años, Gualfín)

Las consecuencias a largo plazo de este efecto acumulativo de episodios no tratados son diversos malestares que se reiteran a medida que avanza la edad: "ya sos persona más grande, te recaidás de cualquier cosa porque estás delicada, más joven no sentís nada" (EG, 41 años, Gualfín).

Por último, las referencias a la etiología de la matriz o pulso como otra dolencia pasible de afectar a las mujeres durante el embarazo y más allá de él, conjugan de manera similar el riesgo de enfermar con situaciones derivadas de las responsabilidades y actividades rutinarias de las mujeres y el apoyo social disponible. La matriz se manifiesta a través de síntomas orgánicos como dolores, palpitaciones, puntadas, hinchazón en la zona abdominal, vómitos, inapetencia ${ }^{(39)}$. A ellos se suman síntomas emocionales, que se refieren como decaimiento, cansancio general, sentirse "desvalorizada", "decaída", estar "sin fuerzas" y/o "sola".

Si bien se plantea que solo las mujeres en la etapa reproductiva pueden "sufrir de la matriz", los hombres adultos pueden sufrir del pulso o padrón, dolencia análoga, cuando están expuestos a esfuerzos o labores ligadas a la agricultura y/o cuidado de la hacienda. Es decir que ambas categorías serían variantes de una misma dolencia (pulso) que puede afectar a hombres y mujeres adultos/as que realizan tareas que suponen un esfuerzo excesivo, denominado "mala fuerza". Los niños, al no estar involucrados en este tipo de tareas, no presentan riesgo de padecer estos problemas.

...dicen que tenemos el hombre y la mujer... los hombres que tienen padrón y las mujeres tienen la matriz, y eso les Ilaman pulso... y dice que eso se corrió, se hunde esa palita así... y hace como si latiera... porque dicen que la matriz cuando uno se ha falseado dice que la matriz se corre, se corre para acá, se sube bien arriba, bien arriba, y le agarra vómito, decaimiento, dolor, y dicen que es porque tiene la matriz subida. (MV, 36 años, Gualfín)

En el caso de las mujeres, hacer mala fuerza durante el embarazo puede derivar en diversos problemas para ellas y el niño en gestación, así como desencadenar partos difíciles.

...hacía fuerza con los tachos y con la leña... me sabía gustar juntar leña y [su mami] ahí me decía ¡no! Te jodés la matriz y después me dolía acá [entre el pecho y el estómago] y no era de comer nada porque ya todo lo volvía, tenía náusea y ya lo volvía todo [los médicos campesinos] dicen que se falsea la matriz, fui al hospital y no me han hallado nada... en los análisis que me hicieron no ha salido nada... y después si, cada semana 
así decaída... que ya quería alzar algo y no tenías fuerza digamos, me cura generalmente con el alcohol y me refrejaba y después de esos tres días que he ido estaba bien pero él ya me dijo "vos vas a tener problemas, cuando tengas un hijo porque es muy chiquita tu matriz y te has falseado" me decía y sí, en el parto de ella... estuve desde las tres de la tarde ¡hasta las once!, después me hicieron la cesárea. (EL, 21 años, Santa Rosa)

Si bien la mala fuerza y el falseado son referidas también como enfermedades, pudiendo esta última afectar a niños, parecería atribuirse a ellas el origen de las dolencias previamente mencionadas ${ }^{(52,53,54)}$.

La medida preventiva central es el fajado en la zona abdominal. Este, junto con la refregada y la manteada, constituyen las únicas prácticas terapéuticas efectivas, orientadas a "acomodar" (reubicar) y contener los órganos internos.

...duele el estómago... tiene una cosita del estómago, bien al centro, es una cosa chiquita... eso está para un lado porque no está donde tiene que estar... entonces dicen que es cuando hacen algo de mucha fuerza, hacen mucha fuerza y se les desubica eso [los médicos campesinos] curan el pulso y refriejan, pero si iba al médico [biomédico] el dolor de estómago no le paraba... le tienen que hacer refrejar dos o tres veces y tiene que usar faja. [Le] agarra a los hombres y a las mujeres, a los chicos no porque ¿qué fuerza van a estar haciendo? A los grandes ya que están con la hacienda [...] yo cuando estaba embarazada ya no me dejaban ir a trabajar en el rastrojo [pero] iyo tengo que hacer mis cosas! A veces, ella me pagaba algún peón para que me trabaje en el rastrojo... me pagaba el peón para que me ayude. (MV, 36 años, Gualfín)

...la matriz le llamamos nosotros, vos vas al hospital, no te descansa, te duele el estómago, estas desvalorizada, tenés sueño [...] tenés mal la matriz, se corre de lugar dicen. Y él [médico campesino] te la ubica bien y te compone, ¿no? te pueden refregar. (NM, 34 años, Molinos)

\section{DISCUSIÓN Y CONCLUSIONES}

En este trabajo intentamos mostrar la relación entre espiritualidad y salud a propósito del análisis de relatos sobre padecimientos que, en mayor o menor medida, suponen un desequilibrio entre la persona y su entorno. En primer lugar, los relatos aquí analizados muestran claramente que el riesgo de asustarse, aicarse, recaidarse o sufrir de la matriz se vincula estrechamente con la ruptura de tabúes ${ }^{(39,55,56,57)}$, que encuentran sustento en las creencias y prácticas religiosas.

En segundo lugar, estos relatos tratan sobre episodios de enfermedad cuyo origen y consecuencias en las trayectorias de salud de las mujeres y sus hijos son interpretadas a la luz de experiencias vitales que dan cuenta del papel del entorno social no solo en la explicación etiológica, sino también en la búsqueda de alternativas terapéuticas. El entorno puede ser el origen de un problema pero, al mismo tiempo, allí se encuentran los actores y recursos a los que se apela para curar la enfermedad, buscar apoyo y sostén emocional durante el proceso. Siguiendo a Price ${ }^{(48)}$, quien ha estudiado las formas culturales de afrontar la enfermedad en comunidades andinas, el conjunto de las creencias religiosas sobre las amenazas y las posibilidades de cura, se recrean en relatos y prácticas compartidos de forma intergeneracional. Esta autora remarca que creencias y rituales religiosos son reinterpretados y adaptados por los individuos en función de sus experiencias vitales, que incluyen habilidades personales y apoyo social, sobre la base de un consenso colectivo. Las dolencias aquí analizadas son expresivas de esta situación, ya que dan cuenta de un proceso de negociación en el que las decisiones individuales se conjugan con las opciones disponibles. La apelación a modelos culturales se justifica en tanto se 
trata de situaciones traumáticas novedosas para las que no se encuentra una respuesta eficaz, lo que resignifica modos de resolver la enfermedad cuya eficacia está legitimada, tanto por la propia experiencia como por la de aquellos cuya palabra es valorada: las mamis y los médicos campesinos. En este sentido, las creencias médicas tradicionales, por su eficacia práctica y simbólica ${ }^{(47)}$, ofrecen a las mujeres alternativas dentro de su entorno social cercano. Asimismo, suponen una menor dependencia de la biomedicina, cuyas opciones no siempre resultan eficaces, accesibles y/o aceptables. En este sentido, si bien se acude a los profesionales biomédicos, son los agentes del entorno doméstico los que intervienen en el cuidado de la salud, lo que remite tanto a parientes y expertos como a deidades y espíritus que habitan el mismo ambiente que el afectado ${ }^{(34)}$. Nuevamente, siguiendo a Price ${ }^{(48)}$, los modos culturalmente aceptables de afrontar los padecimientos van más allá de las habilidades personales a nivel psicológico y remiten a la habilidad para movilizar recursos y apoyo del entorno social.

En tercer lugar, nuestro análisis subraya los sentidos que las mujeres les otorgan a estos padecimientos apelando a experiencias vitales, vínculos y entornos, para los cuales las explicaciones etiológicas basadas exclusivamente en factores orgánicos o psicológicos se revelan como insuficientes. Los testimonios analizados nos permiten plantear que las categorías de susto, recaída y matriz condensan un conjunto de sentidos asociados con la manifestación de un estado emocional negativo que, de prolongarse en el tiempo, torna vulnerables a mujeres y niños. Junto con los síntomas orgánicos, los emocionales ocupan un rol central en la etiología y en la elección de tratamientos ${ }^{(47,58)}$.

En la narrativa sobre susto aparecen menciones a los nervios y a la pena, relación que ya había sido notada por $\operatorname{Crivos}^{(34,36)}$, lo que justifica la inclusión de esta categoría dentro del conjunto de enfermedades del espíritu, ya que tiene consecuencias a nivel espiritual, al afectar la condición misma de persona en términos de valores y expectativas culturales. Las enfermedades del espíritu también comprometen el bienestar del grupo doméstico, ya que supone la imposibilidad de que el niño o adulto que deviene asustado pueda llevar a cabo las actividades y asumir las responsabilidades que se esperan de él. Si bien hombres y niños pueden asustarse, en este trabajo focalizamos en las referencias en las que el susto se deriva de la combinación de situaciones estresantes recurrentes y sucesos repentinos que afectan a las mujeres en condición de susceptibilidad. El aspecto central en la etiología de la matriz es su relación con la mala fuerza como consecuencia de actividades que deben asumir las mujeres durante el embarazo y puerperio cuando no cuentan con el apoyo necesario. Así, las referencias conjugan esfuerzo físico, incumplimiento de tabúes y la inadecuación de la situación de la mujer a sus expectativas, revelando la percepción de una amenaza a ciertos proyectos de vida, a la estabilidad de las relaciones sentimentales y a la subsistencia y bienestar propio y de los hijos. Al respecto, las posibilidades de sufrir algunos de estos padecimientos se asocian a etapas de la vida donde la vulnerabilidad es mayor, sumada a la carga de responsabilidad femenina percibida en el cuidado familiar ${ }^{(47,48,59)}$. Este aspecto puede reconocerse como un marcador de identidad femenina que se sostiene a través de las generaciones, a pesar de los cambios en las actividades y las nuevas oportunidades de trabajo y educación. En el discurso de las mujeres se valorizan la propia autonomía y responsabilidad en la toma de decisiones y el enfrentamiento de diversos problemas relacionados con la crianza de los hijos. Las referencias a la participación de la pareja o esposo durante el embarazo y parto suelen ser mínimas y se relacionan fundamentalmente con el sostén económico ${ }^{(21)}$. En estas circunstancias, destacan el sostén y la asistencia provistos por las mujeres mayores o pares de la unidad doméstica, no solo en momentos críticos de la trayectoria vital -como el embarazo y nacimiento de los hijos- sino también en el desarrollo de las actividades cotidianas. Esta dinámica ha sido analizada por estudios realizados en otras comunidades del noroeste $\operatorname{argentino}^{(60,61)}$. 
Al enmarcarse dentro de categorías médicas tradicionales, estos padecimientos constituyen una forma de expresión de emociones legitimadas culturalmente. En este sentido, referencias a la condición femenina como marcada por el sacrificio y el sufrimiento (ser curtida, sufrida, corajuda) identificadas en los relatos de mujeres ancianas, contrastan con las expresiones de las jóvenes, que si bien no necesariamente asumen estas características como destacables del "ser mujer", se identifican con ellas en algún momento de su trayectoria vital. Así, la circunstancia de atravesar un embarazo sin compañero o sin su apoyo para la crianza de los hijos es una de las más comúnmente asociadas a estados emocionales que predisponen al susto.

En cuarto lugar, la emergencia del susto es explicada como un desequilibrio entre espíritu/cuerpo derivado de una situación traumática o estresante. Varios autores coinciden en que este solo aparecerá en situaciones que los individuos consideren estresantes en cada contexto, variando sus manifestaciones según formas culturales de afrontar situaciones adversas y las expectativas de roles en dichos $\operatorname{contextos}^{(34,62,63,64,65,66)}$. Cuando las mujeres se refieren a sí mismas, el susto -si no es tratado oportunamente- puede derivar en otros problemas de salud de variable intensidad y gravedad para ellas y/o sus hijos, dependiendo de la co-ocurrencia de otros factores como cambios en las rutinas cotidianas y/o eventos estresantes de diversa índole ${ }^{(20,21,27,30)}$. En las explicaciones etiológicas brindadas por ellas, la posibilidad de que un niño sea asustado revela un desajuste entre su conducta y las expectativas y prácticas de las cuidadoras. Más allá del componente de imprevisibilidad en su etiología, las consecuencias del susto y sus variantes a largo plazo se atribuyen a la negligencia de las cuidadoras, lo que pone de relieve lo que se espera de las madres: evitar intencionalmente situaciones que pongan en riesgo la salud, estar atenta a la conducta de los niños, procurar tratamiento inmediato. Al mismo tiempo, subraya las expectativas en torno a la conducta y competencias de los niños, y sus implicancias para su desarrollo a futuro. Ambos tipos de representaciones configuran un elemento central del nicho de desarrollo, presentando variaciones interculturales como ha sido analizado por Super et al. ${ }^{(67)}$. De este modo, las diferencias en el temperamento y comportamiento infantil pueden ser explicadas en términos de salud/ enfermedad y, en este sentido, tales explicaciones se revelan como un mecanismo de control social con implicancias prácticas y morales ${ }^{(30)}$.

En quinto lugar, las categorías de enfermedad aquí analizadas se incluyen dentro del ámbito de la "medicina tradicional" de la región andina ${ }^{(34)}$, y han sido abordados desde los enfoques clásicos de la etnomedicina, como un conjunto de categorías vernáculas discretas ${ }^{(14,68)}$. No obstante, como señala Tousignant $^{(47)}$ para el caso de la pena, el susto remite a estados más amplios que una dolencia específica, y resulta difícil aislarlo de otros síntomas y estados emocionales simultáneos. Estos se articulan en un discurso que los coloca alternativamente en el lugar de consecuencias o de factores que aumentan la susceptibilidad de asustarse y, con ello, desencadenar otros conjuntos de síntomas.

Resulta entonces necesario superar el abordaje esencialista que prima en los enfoques taxonómicos, para dar cuenta de vivencias e itinerarios concretos asociados a los episodios de enfermedad. Esto supone optar por una aproximación pragmatista de los sentidos asignados a estas experiencias en función de condiciones contextuales específicas y atendiendo a las variaciones interindividuales ${ }^{(34)}$. Los relatos aquí analizados dan cuenta de un proceso de atribución de sentidos y valores a síntomas y diagnósticos que varían según estas condiciones contextuales y las formas en que cada narradora interpreta sus experiencias vitales. Sobre esta base, justifican la apelación a diferentes actores y recursos, modificando el curso de la secuencia terapéutica, lo que nos conduce a enfocar en las dimensiones procesuales involucradas en las experiencias de enfermedad, que permiten dar cuenta del rol activo de las mujeres en la búsqueda de alternativas para comprender y resolver sus problemas de salud. Se trata, en definitiva, de un proceso 
reflexivo en el cual se construyen y descartan hipótesis, se buscan nuevos diagnósticos y se implementan las acciones adecuadas para la restitución de la salud.

\section{AGRADECIMIENTOS}

Los datos presentados aquí derivan del Proyecto 11/N821 financiado por la Universidad Nacional de La Plata "Etnografía y redes sociales aplicadas al estudio de las estrategias de manejo del medio ambiente en dos comunidades de Argentina", dirigido por Laura Teves y Carolina Remorini; de la investigacion aprobada y financiada por el Consejo Nacional de Investigaciones Científicas y Técnicas (CONICET) a Carolina Remorini como miembro de la carrera de investigador científico; así como de la investigación enmarcada en la beca doctoral de Maria Laura Palermo financiada por el mismo organismo. Asimismo, del Proyecto de Investigación Plurianual 0270 del Consejo Nacional de Investigaciones Científicas y Técnicas (CONICET) denominado: "Trayectorias, actividades y redes sociales emergentes: Productividad del uso de 'métodos mixtos' en la caracterización de las estrategias de vida en la región del NOA". Deseamos agradecer a las mujeres de Molinos, por su calidez y hospitalidad, por su interés y colaboración en nuestra investigación. Asimismo, a las autoridades municipales, eclesiásticas y a directivos y personal del Hospital Fernández, por su permanente colaboración y facilitación de condiciones para la realización de nuestro trabajo de campo.

\section{REFERENCIAS BIBLIOGRÁFICAS}

1. Krmpotic CS, Saizar MM, editoras. Políticas socio-sanitarias y alternativas terapéuticas: intersecciones bajo la lupa. Buenos Aires: Espacio Editorial; 2016.

2. United Nations, World Health Organization. Official Records of the World Health Organization; Summary report on proceedings minutes and final acts of the International Health Conference [Internet]. No. 2. New York: United Nations, World Health Organization; 1948 [citado 28 jun 2017]. Capítulo 2; p. 100. Disponible en: https:// tinyurl.com/y8dawz8u.

3. Saizar MM. De la censura a la complementariedad domesticada: reflexiones en torno a los modos de vinculación de la biomedicina con otras medicinas. In: Krmpotic CS, Saizar MM, editoras. Políticas socio-sanitarias y alternativas terapéuticas: intersecciones bajo la lupa. Buenos Aires: Espacio Editorial; 2016. p. 25-42.

4. Taussig M. Shamanism, colonialism and the wild man: a study in terror and healing. Chicago: University of Chicago Press; 1987.

5. Kleinman A. The illness narratives: suffering, healing and the human conditions. New York: Basic Books; 1988.

6. Douglas M. Estilos de pensar: ensayos críticos sobre el buen gusto. Barcelona: Gedisa; 1998.

7. Good C. Ethnomedical systems in Africa: patterns of traditional medicine in rural and urban Kenya. New York: The Guilford Press; 1987.

8. Idoyaga Molina A. La selección y combinación de medicinas entre la población campesina de San Juan (Argentina). Scripta Ethnologica. 1999;21:7-33

9. Idoyaga Molina A, Sarudiansky M. Las medicinas tradicionales en el noroeste argentino. Reflexiones sobre tradiciones académicas, saberes populares, terapias rituales y fragmentos de creencias indígenas. Argumentos. 2011;24(66):315-335.

10. Rivers WHR. Medicine, magic and religion. New York: Harcourt; 1924.

11. Clements FE. Primitives concepts of disease. American Archeology and Ethnology. 1932;32(2): 185-252.

12. Ackernecht EH. Primitive medicine and culture pattern. Bulletin of the History of Medicine. 1942;12:545-574.

13. Hughes C. Ethnomedicine. In: Sills DL, editor. International Encyclopedia of the Social Sciences. Vol. 10. New York: Free Press, Macmillan; 1968. p. 87-93.

14. Foster G. Disease etiologies in Non-Western medical systems. American Anthropologist. 1976; 78(4):773-782. 
15. Crivos M, Martínez MR, Pochettino ML. Phytotherapy applied to the treatment of cultural bound syndromes in Calchaqui Valley (Salta, Argentina). In: Gupta RK. Vol. 234. Medicinal and aromatic plants. New Delhi: CBS; 2010. p. 35-45.

16. Drovetta RI. Biomedicina y políticas de salud reproductiva en un contexto rural indígena de la Puna jujeña, Argentina. Barbarói. 2009;31:139154.

17. Drovetta RI. "Antarca no" (de espaldas no): cambios generacionales en la atención del embarazo y el parto en las mujeres de Susques. En: Benedetti A, Tomasi J, compiladores. Espacialidades altoandinas; nuevos aportes desde la Argentina: interacciones con el "mundo de afuera". Buenos Aires: Facultad de Filosofía y Letras; 2014. Tomo 2. p. 195-239.

18. Hirsch S. Salud pública y mujeres indígenas del Noroeste Argentino: las múltiples prácticas de las guaraníes y la atención a la salud reproductiva. En: Cardoso M, Langdon E, editores. Políticas comparadas em saúde indígena na América Latina. Florianópolis: EdUFSC; 2015. p. 169-192.

19. Hirsch S. Moa, inyecciones y pastillas; prácticas y significados en torno a los medicamentos en comunidades guaraníes del norte argentino. En: Hirsch S, Lorenzetti M, editores. Salud pública y pueblos indígenas en la Argentina; encuentros, tensiones e interculturalidad. Buenos Aires: UNSAM Edita; 2016. p. 231-252.

20. Remorini C, Crivos M, Martínez MR, Aguilar Contreras A, Jacob A, Palermo ML. Aporte al estudio interdisciplinario y transcultural del "susto": una comparación entre comunidades rurales de Argentina y México. Dimensión Antropológica. 2012;54:89-126.

21. Remorini C, Morgante MG, Palermo ML. "Mamis" y "guaguas": algunas consideraciones acerca de la maternidad y la crianza a través de las generaciones en Molinos (Salta, Argentina) [lnternet]. Red de Bibliotecas Virtuales de CLACSO; 2010 [citado 20 jun 2017]. Disponible en: https:// tinyurl.com/y7n7cmsk.

22. Jacob A, Palermo ML, Remorini C. Experiencias compartidas, trayectorias singulares; embarazo, parto y puerperio en Molinos (Salta) [Internet]. Buenos Aires: X Congreso Argentino de Antropología Social; 2011 [citado 20 jun 2017]. Disponible en: https://tinyurl.com/y8x9he43.

23. Palermo ML. La atención de la salud materno infantil desde las perspectivas de la población y del personal sanitario en Molinos (Salta, Argentina). In: Castro Esnal A, Funes ML, Grosso
M, Kuperszmit N, Murgo A, Romero G, editoras. Entre pasados y presentes IV: estudios contemporáneos en ciencias antropológicas. Buenos Aires: Asociación Amigos del Instituto Nacional de Antropología; 2014. p. 165-182.

24. Remorini C. Trayectorias de desarrollo infantil en contexto ecológico; estudio etnográfico del cuidado, la crianza y la participación de los niños/ as en actividades cotidianas en comunidades rurales e indígenas. [Proyecto Carrera de Investigador Científico]. Buenos Aires: CONICET; 2015.

25. Remorini C, Palermo ML. Es que antes no sabía haber doctor; alternativas para el cuidado de la salud materno-infantil en una población de los Valles Calchaquíes (Salta, Argentina) [Internet]. Viena: $54^{\circ}$ Congreso Internacional de Americanistas; 2012 [citado 20 jun 2017]. Disponible en: https://tinyurl.com/yb87vg3b.

26. Remorini C. Childrearing and the shaping of children's emotional experiences and expressions in two Argentinian communities. Global Studies of Childhood. 2012;2(2):144-157.

27. Remorini C. Estudio etnográfico de la crianza y la participación infantil en comunidades rurales de los Valles Calchaquíes Septentrionales (Noroeste argentino). Bulletin de I'Institut Français d'Études Andines. 2013;42(3):411-433.

28. Remorini C, Palermo ML. Salud materno-infantil y políticas públicas para Pueblos Originarios. Reflexiones a partir de una investigación etnográfica. In: Cardoso M, Langdon E, editores. Políticas Comparadas em Saúde Indígena na América Latina. Florianopolis: EdUFSC; 2015. p. 247-278.

29. Remorini C, Palermo ML. Acerca de "niños en riesgo" y "padres no suficientemente buenos". Conceptualizaciones en torno a la infancia, los niños y sus familias como objeto de políticas de salud en Molinos (Salta, Argentina) [Internet]. En: Olarte MR, compiladora. Infancias y juventudes latinoamericanas. Manizales: Centro Editorial CINDE, Centro de Estudios Avanzados en Niñez y Juventud, Fundación Centro Internacional de Educación y Desarrollo Humano, Universidad de Manizales, Buenos Aires-CLACSO; 2015 [citado 20 jun 2017]. p. 100-111. Disponible en: https:// tinyurl.com/y8kpa72f.

30. Remorini C, Palermo ML. "Los míos... ¡todos asustados!": vulnerabilidad infantil y trayectorias de desarrollo en los Valles Calchaquíes Salteños. Mitológicas. 2016;XXXI:83-112.

31. Remorini C, Morgante MG. Las relaciones intergeneracionales y las prácticas de cuidado en el seno de unidades domésticas vallistas del De- 
partamento de Molinos (Salta, Argentina). Buenos Aires: XII Jornadas Nacionales de Debate Interdisciplinario en Salud y Población; 2016.

32. Palermo ML. Estudio etnográfico de los itinerarios terapéuticos en la atención de la salud materna e infantil en Molinos (Salta, Argentina). [Tesis de doctorado]. La Plata: Universidad Nacional de La Plata; 2016.

33. Argentina, Instituto Nacional de Estadística y Censos. Censo Nacional de Población, Hogares y Viviendas 2010 [Internet]. Buenos Aires: Instituto Nacional de Estadística y Censos; 2010 [citado 20 jun 2017]. Disponible en: https://tinyurl.com/ ybyax $96 z$.

34. Crivos M. Contribución al estudio antropológico de la medicina tradicional de los Valles calchaquíes (Salta, Argentina). [Tesis de doctorado]. La Plata: Universidad Nacional de La Plata; 2003.

35. Crivos M, Martínez MR, Remorini C, Teves L, Morgante MG. El agua en la narrativa sobre las estrategias de vida en los Valles Calchaquíes Salteños. Testimonios. 2017;(6):100-124.

36. Crivos M. Algunos aspectos de la medicina popular en Molinos (provincia de Salta). Sapiens. 1978;2:15-27.

37. Crivos M, Eguía AC. Dos estudios antropológicos sobre medicina tradicional de los Valles Calchaquíes, Argentina. Folklore Americano. 1981;31:71-76.

38. Crivos M, Martínez MR. Una propuesta metodológica para el relevamiento de información en el dominio de la etnobiología; las estrategias frente a la enfermedad en el contexto de la "unidad doméstica". In: Contribuciones a la Antropología Física Latinoamericana: memoria del IV Simposio de Antropología Física "Luis Montané". México: Instituto de Investigaciones Antropológicas, Universidad de La Habana; 1996. p. 99-104.

39. Martínez MR, Pochettino ML. Análisis de los recursos terapéuticos utilizados en el Valle Calchaquí; las mujeres dicen acerca de dolencias y "remedios". Relaciones de la Sociedad Argentina de Antropología. 2004;29:163-182.

40. Martínez MR, Crivos M. About the hospital and other medical alternatives in Molinos' everyday life. Praga: XVI International Oral History Conference; 2010.

41. Crivos M. Martínez MR. La aproximación etnográfica al estudio de la medicina tradicional: una experiencia en los Valles Calchaquíes Salteños (Argentina) [Internet]. Quito: 49 Congreso Inter- nacional de Americanistas; 1997 [citado 20 jun 2017]. Disponible en: https://tinyurl.com/ydavxlvd.

42. Harkness S, Super C. The developmental niche: a conceptualization at the interface of child and culture. International Journal of Behavioral Development. 1986;9(4):545-569.

43. Elder GH, Rockwell R. The life-course and human development: an ecological perspective. International Journal of Behavioral Development. 1979;2(1):1-21.

44. Bronfenbrenner U. La ecología del desarrollo humano. Experimentos en entornos naturales y diseñados. 1a ed. Barcelona: Paidós; 1987.

45. Crivos M, Martínez MR. Aspectos de la percepción de algunos fenómenos meteorológicos y naturales entre los pobladores de Molinos (Salta, Argentina). En: Goloubinoff M, Katz E, Lammel A, compiladores. Antropología del clima en el mundo hispanoamericano. Tomo II. Quito: Ediciones Abya-Yala; 1997. p. 135-152.

46. La Riva González P. De I'enfant rêvé à I'enfant construit: représentations de la petite enfance dans une communauté des Andes du sud du Pérou. In: Bonnet D, Suremain CE, Rollet C, editores. Modèles d'enfances: successions, transformations, croisements. París: Éditions des Archives Contemporaines; 2012.

47. Tousignant M. Pena in the Ecuadorian Sierra: a psychoanthropological analysis of sadness. Culture, Medicine and Psychiatry. 1984;8(4):381-398.

48. Price L. Metalogue in coping with illness: cases from Ecuador. Qualitative Health Research. 1992; 2(2):135-158.

49. Idoyaga Molina A. El simbolismo de lo cálido y lo frío; reflexiones sobre el daño, la prevención y la terapia entre los criollos de San Juan (Argentina). Mitológicas. 1999;14(1):7-27.

50. Fernández Juárez G. Cirugía y pueblos amerindios: un reto intercultural. Revista Peruana de Medicina Experimental y Salud Pública. 2010;27(1):102-113.

51. La Riva González P. Le walthana hampi ou la reconstruction du corps; conception de la grossesse dans les Andes du Sud du Pérou. Journal de la Société des Américanistes. 2000;86:169184.

52. Crivos M, Eguía A. Un aspecto de la medicina tradicional de los Valles Calchaquíes. La Semana Médica. 1980;157(5):199-202. 
53. Palma, NH. Estudio antropológico de la medicina popular de la Puna argentina. Buenos Aires: Cabargón; 1973.

54. Bianchetti MC. Cosmovisión sobrenatural de la locura. Pautas populares de salud mental en la Puna argentina. 6a ed. Salta: Hanne; 1996.

55. Katz E. Recovering after childbirth in the Mixtec highlands (México) [Internet]. Heidelberg: 11 Conférence Internationale d'Ethnomédecine; 1993 [citado 20 jun 2017]. Disponible en: https:// tinyurl.com/y7p266pg.

56. Platt T. O feto agressivo; parto, formação da pessoa e mito-história nos Andes. Tellus. 2009;9(17):61-109.

57. Douglas M. Pureza y peligro; un análisis de los conceptos de contaminación y tabú. Buenos Aires: Nueva Visión; 2007.

58. Baer RD, Weller SC, García de Alba García J, Glazer M, Trotter R, Pachter L, Klein RE. A cross-cultural approach to the study of the folk illness nervios. Culture, Medicine and Psychiatry. 2003;27(3):315-337.

59. Pribilsky J. Nervios and 'modern' childhood: migration and changing contexts of child life in the Ecuadorian Andes. Childhood. 2001;8(2):251273.

60. Hirsch S. Maternidad, trabajo y poder: cambios generacionales en las mujeres guaraníes del norte argentino. In: Hirsch S, coordinadora. Mujeres indígenas en la Argentina; cuerpo, trabajo y poder. Buenos Aires: Biblos; 2008. p. 231-251
61. Drovetta RI. Intervenciones de la salud pública en los procesos reproductivos de mujeres indígenas de la Puna argentina. En: Hirsch S, Lorenzetti $M$, editores. Salud pública y pueblos indígenas en la Argentina; encuentros, tensiones e interculturalidad. Buenos Aires: UNSAM Edita; 2016. p. 51-70.

62. Sayres W. Status transition and magical fright. América Indígena. 1955;15(4):292-300.

63. Rubel A. The epidemiology of a folk illness: susto in Hispanic America. Ethnology. 1964;3(3):268-283.

64. Trotter R. Susto: the context of community morbidity patterns. Ethnology. 1982;21(3):215-226.

65. Signorini I. Patterns of fright: multiple concepts of susto in a Nahua-Ladino community of the Sierra de Puebla (México). Ethnology. 1982;21(4):313-323.

66. Zolla C, editor. La medicina tradicional de los pueblos indígenas de México. México DF: Instituto Indigenista Interamericano; 1994.

67. Super CM, Axia G, Harkness S, WellesNyström B, Zylicz PO, Parmar P, Bonichini S, et al. Culture, temperament, and the "difficult child": a study in seven western cultures. European Journal of Developmental Science. 2008;2(1-2):136-157.

68. Conklin H. Lexicographical treatment of folk taxonomies. International Journal of American Linguistics. 1962;28(2)4:119-141.

\section{FORMA DE CITAR}

Remorini C, Palermo ML, Schvartzman L. Espiritualidad y salud: problemas de salud durante el embarazo y el puerperio y sus consecuencias en las trayectorias de mujeres y niños (Salta, Argentina). Salud Colectiva. 2018;14(2):193-210. doi: $10.18294 /$ sc. 2018.1506.

Recibido: 6 de julio de 2017 | Versión final: 22 de noviembre de 2017 | Aprobado: 30 de diciembre de 2017 